\title{
Dielectric properties of composite based on ferroelectric copolymer of poly(vinylidene fluoride-trifluoroethylene) and ferroelectric ceramics of barium lead zirconate titanate
}

\author{
A. V. Solnyshkin*, ${ }^{*}$, I. L. Kislova*, I. M. Morsakov*, A. N. Belov ${ }^{\dagger}$, \\ V. I. Shevyakov ${ }^{\dagger}$ D. A. Kiselev* and V. V. Shvartsman $\$$ \\ *National Research University of Electronic Technology \\ Zelenograd 124498, Moscow, Russia \\ ${ }^{\dagger}$ Department of Condensed Matter Physics \\ Tver State University, Tver 170100, Russia \\ "National University of Science and Technology "MISiS" \\ 119049 Moscow, Russia \\ $\S_{\text {Institute for Materials Science, University of Duisburg-Essen }}$ \\ Essen D 45141, Germany \\ Ta.solnyshkin@mail.ru
}

Received 17 September 2017; Revised 6 October 2017; Accepted 9 October 2017; Published 31 October 2017

\begin{abstract}
A study of dielectric properties of composite films on the base of poly(vinylidene fluoride-trifluoroethylene) copolymer P(VDF$\mathrm{TrFE}$ ) and ferroelectric ceramics of barium lead zirconate titanate (BPZT) solid solution is presented in this work. The composite films containing up to 50 vol. $\%$ of BPZT grains with size $\sim 1 \mu \mathrm{m}$ were prepared by the solvent cast method. Frequency dependences of real and imaginary components of the complex permittivity were determined. The concentration dependence of the dielectric constant was discussed.
\end{abstract}

Keywords: Dielectric response; ferroelectric polymer; composite film.

\section{Introduction}

Poly(vinylidene fluoride) (PVDF) and related copolymers exhibit a variety of characteristic mechanical and electrical properties, such as piezoelectricity, pyroelectricity, nonlinear optical properties, etc. Therefore, a considerable amount of works has been devoted to PVDF and its copolymers in relation to their notable electro-physical activities. ${ }^{1-3}$ These activities have been shown to a rise from cooperative orientation of molecular dipoles originated from their ferroelectric nature. ${ }^{1}$

Polar polymeric materials possess a number of advantages as compared with crystalline ferroelectrics: low density, mechanical elasticity, stability of physical properties, moisture resistance, easiest and cost effective fabrication, any size and shape sample preparation, etc. At the same time, they are inferior to crystalline ferroelectric materials in some physical parameters such as the value of the pyroelectric coefficient and piezoelectric modules, the magnitude of spontaneous polarization. Therefore, researchers pay attention increasingly to the preparation of materials possessed properties of polar polymer and crystalline ferroelectrics. ${ }^{4-8}$ These materials are composite structures based on polymers and crystalline or ceramic ferroelectrics as fillers in the polymeric matrix. Wide usage of polymer-based ferroelectric composites is motivated by tight balance between dielectric permittivity $(\varepsilon)$ of inclusions (high $\varepsilon$ ) and polymer matrix (low $\varepsilon$ ), i.e., in the composites, high $\varepsilon$ of ceramics grains is compensated by low $\varepsilon$ of polymer. This balance leads to significant figures of merit $p / \varepsilon$ and piezoelectric modulus $g_{i j}$.

Composite materials are multiphase systems, the properties of which depend on the number of phases, their concentration in the material, electrical characteristics of each phase, etc. In general, the composites are heterogeneous materials. Responses of these structures to external actions (electric field, mechanical stress, temperature, etc.) are summary ones determined by responses of the individual components of the systems, or are attributed to some property not inherent to any of the composite components. By now, the attention of many researchers is focused on ferroelectric composites containing the polar polymer (or copolymer) and ferroelectric perovskites like the lead zirconate titanate (PZT), barium titanate and others. ${ }^{9-14}$ These ferroelectric crystal inclusions can significantly increase the dielectric parameters and allow producing the materials with high- $k$ for the dielectric applications. Also, the presence of ceramics in the polymer matrix leads to a significant improvement in

This is an Open Access article published by World Scientific Publishing Company. It is distributed under the terms of the Creative Commons Attribution 4.0 (CC-BY) License. Further distribution of this work is permitted, provided the original work is properly cited. 
pyroelectric and piezoelectric properties. However, for some pyroelectric and piezoelectric applications, low values of dielectric permittivity and losses are required. Experimental data for those composite materials and theoretical concepts of their physical characteristics behavior are quite contradictory.

In our previous works, we prepared the film composite film structures on the base of ferroelectric copolymer of poly (vinylidene fluoride-trifluoroethylene) $\mathrm{P}(\mathrm{VDF}-\mathrm{TrFE})$ and ferroelectric ceramics of barium lead zirconate titanate $\mathrm{Pb}_{0.75} \mathrm{Ba}_{0.25}\left(\mathrm{Zr}_{0.53} \mathrm{Ti}_{0.47}\right) \mathrm{O}_{3} \quad(\mathrm{BPZT})^{15,16}$ and investigated their pyroelectric properties by the dynamic method. ${ }^{17,18}$ The response to the thermal action on ferroelectric composites is determined by both pyroelectric parameters and dielectric characteristics. Dielectric parameters must necessarily be considered for the dynamic range, when the response is excited by the alternating external action. Thus, the purpose of this work was the study of dielectric response of the composite samples. This study is necessary for further analysis of pyroelectric and piezoelectric activities of the P(VDFTrFE) + BPZT composites.

\section{Experimental}

To prepare the samples, the $\mathrm{P}(\mathrm{VDF}-\mathrm{TrFE})$ copolymer was used as the matrix of the composite material. At crystallization of the $\mathrm{P}(\mathrm{VDF}-\mathrm{TrFE})$ copolymer from a solution or melt, the ferroelectric crystalline phase ( $\beta$-phase) was produced without any additional treatment such as the mechanical stretching, annealing and applying the strong electric fields. The ratio of VDF/TrFE in the copolymer is about 72/28. This copolymer has high values of pyroelectric and piezoelectric coefficients and is characterized by high temperature stability of the physical parameters. Barium lead zirconate titanate ceramics powder was chosen as filler of the polymer composite matrix. Barium lead zirconate titanate ceramics are between the ferroelectrically hard and soft ceramics. These ceramics with Ba content $\sim 20 \%$ and $\mathrm{Ti} 40-50 \%$ exhibit excellent piezoelectric and pyroelectric properties and possess low dielectric loss factor. ${ }^{19}$ Dielectric permittivity of BPZT ceramics measured at $1 \mathrm{kHz}$ is nearly 2100 and tangent dielectric losses $\tan \delta \approx 0.01$.

The P(VDF-TrFE) + BPZT composite polymer films were fabricated by the solvent-cast method. A more detailed description of the composite sample preparation was given in Refs. 15 and 16. The ceramic filler content in the films was varied within $10-50$ vol. $\%$. The average grain size of the ceramics was about $1 \mu \mathrm{m} .{ }^{15}$ The thickness of the film samples was dependent on the filler content and was equal to 25-95 $\mu \mathrm{m}$. The virgin film samples did not possess macroscopic pyroelectric and piezoelectric properties. The corona discharge method was used to polarize the composite films. To determine dielectric parameters, aluminium electrodes were sputtered on both surfaces of the unpolarized and polarized composite samples. A diameter of disk-shaped electrodes was equal to $12 \mathrm{~mm}$ and their thickness was about $40 \mathrm{~nm}$.
Dielectric dispersion measurements in the frequency range between $10^{-1}$ and $10^{7} \mathrm{~Hz}$ were carried out with the Novocontrol ALPHA High-Resolution Dielectric Analyzer. The frequency-dependent complex dielectric permittivity $\varepsilon *=$ $\varepsilon^{\prime}-j \varepsilon^{\prime \prime}$ was measured. Here, $\varepsilon^{\prime}$ and $\varepsilon^{\prime \prime}$ are the real and imaginary parts of the complex dielectric permittivity respectively, $j$ is the imaginary unit.

The topographic image of the composite sample surface was obtained by atomic force microscopy (AFM) using a Nanoscope-IIIa microscope (Digital Instruments) with a cantilever rigidity of $15 \mathrm{~N} / \mathrm{m}$ and a resonance frequency of $130 \mathrm{kHz}$. Measurements were carried out in the contact mode; i.e., the cantilever was directly contacted with the film surface.

\section{Results and Discussion}

The AFM measurements revealed two types of structures in the pure $\mathrm{P}(\mathrm{VDF}-\mathrm{TrFE})$ samples which had different contrasts over the film surface (Fig. 1(a)). The dark area in the image corresponds to an amorphous $\alpha$-phase of the copolymer.

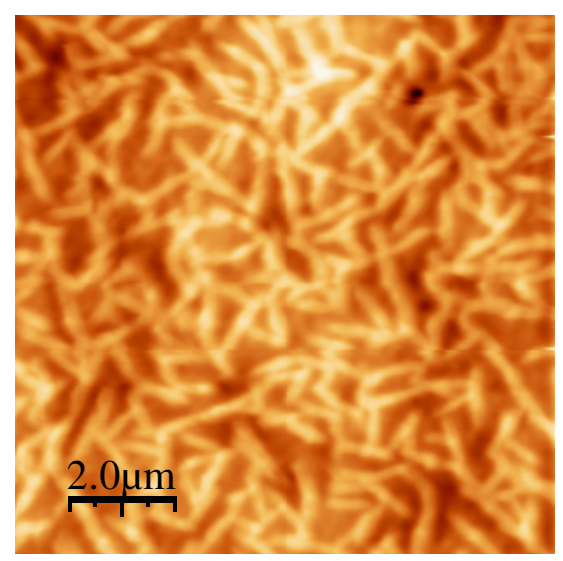

(a)

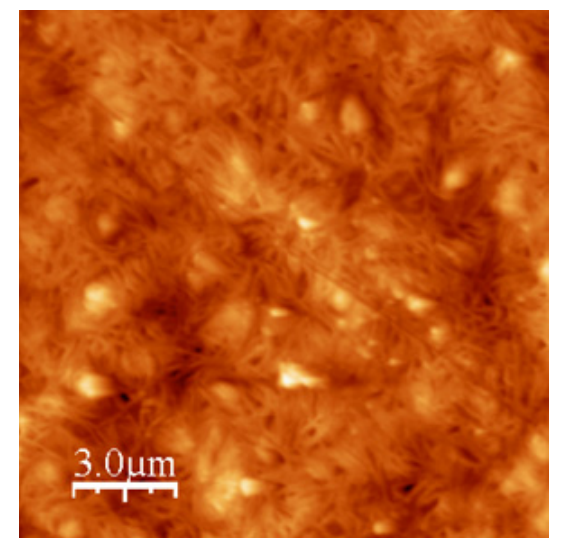

(b)

Fig. 1. Topography images of P(VDF-TrFE) copolymer film (a) and $\mathrm{P}(\mathrm{VDF}-\mathrm{TrFE})+20 \%$ BPZT composite film (b). 


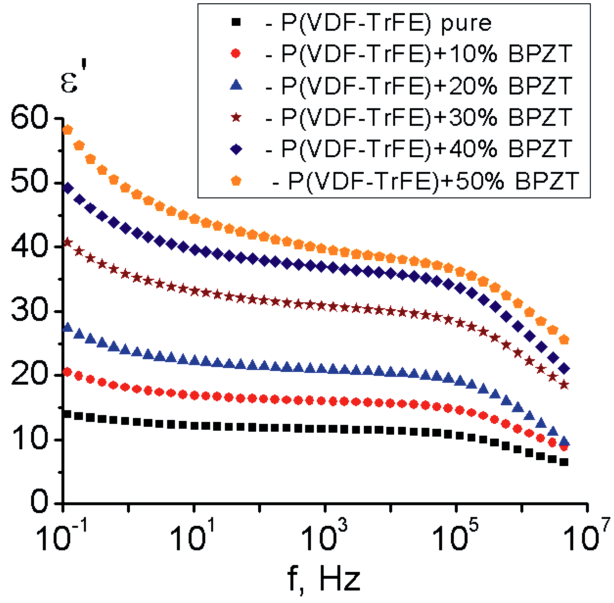

(a)

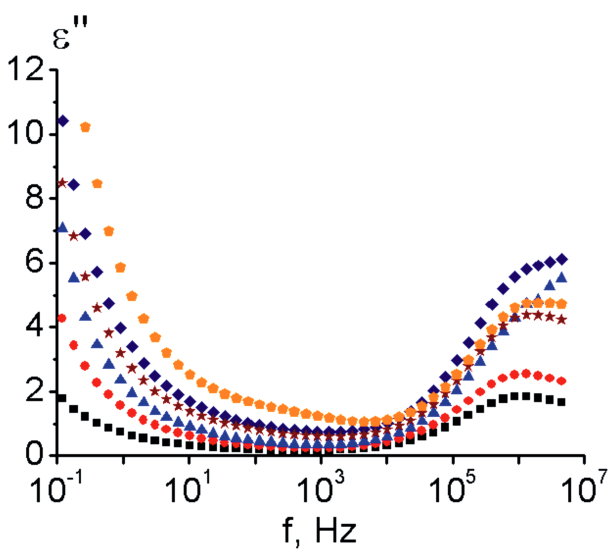

(b)

Fig. 2. The typical frequency dependence of the real (a) and imaginary (b) components of the complex dielectric permittivity of P(VDFTrFE) + BPZT composite films.

Bright domains are attributed to the copolymer crystalline fraction. ${ }^{15}$

The inclusions of ferroelectric ceramic grains lead to changes in the crystalline fraction of the copolymer matrix. In the composite films, an increase of the crystalline phase in the polymer matrix is observed (Fig. 1(b)).

Below, the dielectric measurement results are presented for the virgin and polarized composite films. Sample sets (10 samples of each concentration) were used in this work. The dielectric measurements had been repeatedly performed and the results were well reproducible for each parameter set.

It was found that the dispersion of dielectric parameters of the composite films depended on the level of BPZT ceramics inclusions. Figure 2 shows the dielectric characteristics dispersion determined at the room temperature. As one can see from the curves presented in Fig. 2, the presence of ceramics grains in the $\mathrm{P}(\mathrm{VDF}-\mathrm{TrFE})$ matrix does not significantly change the frequency dependence behavior of $\varepsilon^{\prime}$ and $\varepsilon^{\prime \prime}$, but the dielectric characteristics values in the whole frequency range increase with the ceramic filler growth.

At low frequencies $\left(f<10^{3} \mathrm{~Hz}\right)$, the effect of ferroelectric ceramic grains on the dielectric behavior of composite films is more significant than at the high-frequency range. This is associated with an increase in DC-conductivity at the addition of BPZT ceramic inclusions to the composite polymer matrix. The DC-conductivity was found by the standard method of current-voltage characteristics. Below, we present the conductivity values measured for the composite films P(VDFTrFE) + BPZT:

- $\sigma_{o}=(1.0 \pm 0.3) \cdot 10^{-13} \quad \Omega^{-1} \cdot \mathrm{cm}^{-1}$ (fraction of ceramics -0 vol. \%);

- $\sigma_{o}=(3.2 \pm 1.2) \cdot 10^{-13} \quad \Omega^{-1} \cdot \mathrm{cm}^{-1}$ (fraction of ceramics -10 vol. $\%)$

- $\sigma_{o}=(8.0 \pm 2.3) \cdot 10^{-13} \quad \Omega^{-1} \cdot \mathrm{cm}^{-1}$ (fraction of ceramics -20 vol. \%);
- $\sigma_{o}=(1.9 \pm 0.4) \cdot 10^{-12} \Omega^{-1} \cdot \mathrm{cm}^{-1}$ (fraction of ceramics -30 vol. \%);

- $\sigma_{o}=(4.1 \pm 1.7) \cdot 10^{-12} \quad \Omega^{-1} \cdot \mathrm{cm}^{-1}$ (fraction of ceramics -40 vol. $\%)$;

- $\sigma_{o}=(9.5 \pm 3.5) \cdot 10^{-12} \Omega^{-1} \cdot \mathrm{cm}^{-1}$ (fraction of ceramics -50 vol.\%).

The dielectric constant dependence on the ceramic filler content in the composite material (Fig. 3) was determined on the basis of the dielectric dispersion studies. For such dependence, the model proposed in Ref. 20 describes the experimental data most appropriately. Composite material is considered as a binary system consisting of a continuous matrix with a dielectric constant $\varepsilon_{1}$ and ellipsoidal inclusions with a dielectric constant $\varepsilon_{2}$. According to Ref. 20, the composite dielectric permittivity is described by the relation

$$
\varepsilon=\varepsilon_{1}\left[1+\frac{n q\left(\varepsilon_{1}-\varepsilon_{2}\right)}{n \varepsilon_{1}+\left(\varepsilon_{2}-\varepsilon_{1}\right)(1-q)}\right],
$$

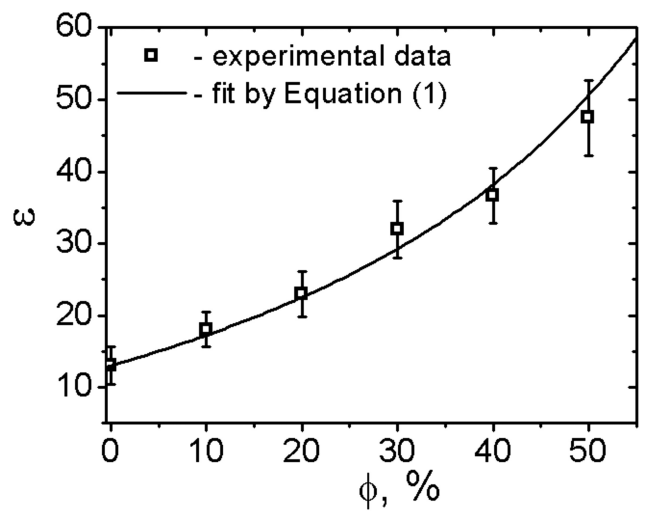

Fig. 3. Dependence of dielectric constant on the percentage of ceramics grains in the $\mathrm{P}(\mathrm{VDF}-\mathrm{TrFE})+\mathrm{BPZT}$ composite films. Measurements were carried out at $f=10^{3} \mathrm{~Hz}$. 
where $q$ is volumetric fraction of ellipsoidal particles in the composite $(0 \leq q \leq 1), \phi=q \cdot 100 \%, \phi$ is a percentage of inclusions, $n$ is a parameter determined by the inclusion shapes. For the spherical particles embedded into the matrix, $n=3$.

Approximation of the experimental dependence of the dielectric permittivity on the volumetric fraction of ceramics inclusions in the $\mathrm{P}(\mathrm{VDF}-\mathrm{TrFE})+\mathrm{BPZT}$ composite was carried out at values $\varepsilon_{1}=12$ and $\varepsilon_{2}=2100\left(f=10^{3} \mathrm{~Hz}\right)$ as well as the parameter $n=3$. As seen from the dependence presented in Fig. 3, the experimental data follow Eq. (1) with these fitting parameters. This indicates that the shape of the BPZT ceramic inclusions in the polymer matrix is close to spherical. It is necessary to note that at $n=3$, Eq. (1) is transformed into the Maxwell-Garnett expression describing the dielectric response of a binary composite system within the effective medium theory. ${ }^{21}$ This model describes well our experimental data of the percentage dependences of dielectric permittivity in the frequency range from $10^{-1}$ to $10^{7} \mathrm{~Hz}$.

The presented results were obtained for the unpolarized composite samples. Preliminary poling by the electric field exceeded the coercive one $\left(E_{C}=4 \cdot 10^{7} \mathrm{~V} \cdot \mathrm{m}^{-1}\right)$ affects the dielectric behavior of the composite films. The poling was used to induce the unipolar state in the prepared composite films that the pyroelectric and piezoelectric activities were appeared. A corona discharge is a most effective method for inducing the pyroelectric and piezoelectric properties. ${ }^{18}$ The $\mathrm{P}(\mathrm{VDF}-\mathrm{TrFE})+\mathrm{BPZT}$ composite samples polarized by corona discharge exhibit less values of the real and imaginary parts of the complex dielectric permittivity (approximately $20 \%$ lower than for unpolarized samples) as well as for the copolymer samples without the ceramic inclusions. ${ }^{22,23}$

\section{Conclusion}

The dielectric properties of the composite material based on the P(VDF-TrFE) copolymer with the inclusions of barium lead zirconate titanate ceramics were studied. Dependence of the dielectric constant on the ceramic volume fraction was determined using the dielectric dispersion measurements of the composite films with different percentages of the BPZT grains. These dielectric data are necessary to calculate figures of merit (FM) for pyroelectric thermal detectors and infrared imagers operating the dynamic mode in a broad frequency range of heat flux modulation, since the characteristics of pyroelectric functional elements are determined by FM. The dynamic pyroelectric response of the functional elements is considered in a wide frequency range. ${ }^{18}$ The frequency dependence of the dielectric properties are required, as the pyroelectric response is determined by $p / \varepsilon^{\prime}$ or $p / \sqrt{\varepsilon^{\prime \prime}}$, where $p$ is pyroelectric coefficient. It allows to define a frequency dependence of pyroelectric characteristics of the device functional elements. Additionally, the frequency dependences of the dielectric parameters are useful to evaluate the piezoelectric activity of the $\mathrm{P}(\mathrm{VDF}-\mathrm{TrFE})+\mathrm{BPZT}$ composite films since the material is promising for acoustic transducer and sensor applications. To estimate the thermal stability of the electrophysical characteristics of the composite material, further studies are necessary to carry out in wide temperature range including the glass transition and the ferroelectric phase transition of the polymer matrix. Further research is in progress.

\section{Acknowledgment}

This work was supported by the Russian Scientific Foundation (Grant N 15-19-00138).

\section{References}

${ }^{1}$ B.-J. Jungnickel, Ferroelectric Polymers: Chemistry, Physics, and Applications, ed. H. S. Nalwa, (Marcel Dekker, New York, 1995), pp. 233-260.

${ }^{2}$ P. Ueberschlag, PVDF piezoelectric polymer, Sensor Rev. 21, 118 (2001).

${ }^{3}$ K. S. Ramadan, D. Sameoto and S. Evoy, A review of piezoelectric polymers as functional materials for electromechanical transducers, Smart Mater. Struct. 23, 033001 (2014).

${ }^{4}$ K. H. Chew, F. G. Shin, B. Ploss, H. L. W. Chan and C. L. Choy, Primary and secondary pyroelectric effects of ferroelectric 0-3 composites, J. Appl. Phys. 94, 1134 (2003).

${ }^{5}$ M. Dietze, J. Krause, C.-H. Solterbeck and M. Es-Souni, Thick film polymer-ceramic composites for pyroelectric applications, J. Appl. Phys. 101, 054113 (2007).

${ }^{6}$ C. Wu, G. Cai, W. Luo, Q. Peng, X. Sun and W. Zhang, Enhancement of pyroelectric properties of composite thick films using Mn-doped PZT, Sens. Actuators A 199, 24 (2013).

${ }^{7}$ S. Anandhan and S. Bandyopadhyay, Nanocomposites and Polymers with Analytical Methods, ed. J. Cuppoletti, (InTech, 2011), pp. 3-30.

${ }^{8}$ C. Baur, D. J. Apo, D. Maurya, S. Priya, W. Voit, Advances in Piezoelectric Polymer Composites for Vibrational Energy Harvesting, ACS Symp. Ser. 1161, 1 (2014).

${ }^{9}$ A. Jain and K. J. Prashanth et al., Dielectric and piezoelectric properties of PVDF/PZT composites: A review, Polym. Eng. Sci. 55, 1589 (2015).

${ }^{10}$ M. Arbatti, X. Shan and Z. Cheng, Ceramic-Polymer Composites with High Dielectric Constant, Adv. Mater. 19, 1369 (2007).

${ }^{11}$ H. Tang and H. A. Sodano, Ultra High Energy Density Nanocomposite Capacitors with Fast Discharge Using $\mathrm{Ba}_{0.2} \mathrm{Sr}_{0.8} \mathrm{TiO}_{3}$ Nanowires, Nano Lett. 13, 1373 (2013).

${ }^{12}$ Z.-M. Dang, J.-K. Yuan, J.-W. Zha, P.-H. Hu, D.-R. Wang and Z.-Y. Cheng, High-permittivity polymer nanocomposites: Influence of interface on dielectric properties, J. Adv. Dielect. 3, 1330004 (2013).

${ }^{13}$ Y. Wang, J. Cui, Q. Yuan, Y. Niu, Y. Bai and H. Wang, Significantly Enhanced Breakdown Strength and Energy Density in Sandwich-Structured Barium Titanate/Poly(vinylidenefluoride) Nanocomposites, Adv. Mater. 27, 6658 (2015).

${ }^{14}$ X. Zhang, Y. Shen, B. Xu, Q. Zhang, L. Gu, J. Jiang, J. Ma, Y. Lin and C.-W. Nan, Giant Energy Density and Improved Discharge 
Efficiency of Solution-Processed Polymer Nanocomposites for Dielectric Energy Storage, Adv. Mater. 28, 2055 (2016).

${ }^{15}$ A. V. Solnyshkin, D. A. Kiselev, A. A. Bogomolov, A. L. Kholkin, W. Künstler and R. Gerhard, Atomic force microscopy study of ferroelectric films of $\mathrm{P}(\mathrm{VDF}-\mathrm{TrFE})$ copolymer and composites based on it, J. Surf. Invest. X-ray, Synchr. Neutron Techn. 2, 692 (2008).

${ }^{16}$ M. V. Silibin, A. V. Solnyshkin, D. A. Kiselev, A. N. Morozovska, E. A. Eliseev, S. A. Gavrilov, M. D. Malinkovich, D. C. Lupascu and V. V. Shvartsman, Local ferroelectric properties in polyvinylidene fluoride/barium lead zirconatetitanatenanocomposites: Interface effect, J. Appl. Phys. 114, 144102 (2013).

${ }^{17}$ A. V. Solnyshkin, I. M. Morsakov, A. G. Kanareikin and A. A. Bogomolov, Pyroelectric effect in composites based on copolymer P(VDF-TrFE) and ferroelectric ceramics BPZT, Bull. Russ. Acad. Sci. Phys. 74, 1287 (2010).

${ }^{18}$ A. V. Solnyshkin, I. M. Morsakov, A. A. Bogomolov, A. N. Belov, M. I. Vorobiev, V. I. Shevyakov, M. V. Silibin, V. V. Shvartsman, Dynamic pyroelectric response of composite based on ferroelectric copolymer of poly(vinylidene fluoride-trifluoroethylene) and ferroelectric ceramics of barium lead zirconate titanate, Appl. Phys. A. 121, 311 (2015).

${ }^{19}$ G. Li, G. Haertling, Dielectric, ferroelectric and electric fieldinduced strain properties of $\left(\mathrm{Pb}_{1-x} \mathrm{Ba}_{x}\right)\left(\mathrm{Zr}_{1-y} \mathrm{Ti}_{y}\right) \mathrm{O}_{3}$ ceramics, Ferroelectrics 166, 31 (1995).

${ }^{20}$ T. Yamada, T. Ueda and T. Kitayama, Piezoelectricity of a highcontent lead zirconate titanate/polymer composite, Sens. Actuators A 53, 4328 (1982).

${ }^{21}$ T. C. Choy, Effective Medium Theory. Principles and Applications, 2nd edn. (Clarendon Press, Oxford, 2016).

${ }^{22}$ A. V. Solnyshkin, M. Wegener, W. Künstler and R. GerhardMulthaupt, Anomalies of dielectric properties of vinylidene fluoride-trifluoroethylene copolymer films, Phys. Solid State 50, 562 (2008).

${ }^{23}$ A. V. Solnyshkin, I. L. Kislova, M. V. Silibin and D. A. Kiselev, Polarization Effect on Dielectric Response of Ferroelectric Copolymer P(VDF-TrFE), Ferroelectrics 469, 144 (2014). 